\title{
Multipoint Padé-Type Approximants. Exact Rate of Convergence
}

\author{
F. Cala Rodríguez and G. López Lagomasino
}

\begin{abstract}
We study the rate with which sequences of interpolating rational functions, whose poles are partially fi ed, approximate Markov-type analytic functions. Applications to interpolating quadratures are given.
\end{abstract}

\section{Introduction}

Let $\mu$ be a finit positive Borel measure whose support $\operatorname{supp}(\mu)$ is contained in $B=$ $[-1,1]$. Set

$$
\hat{\mu}=\int \frac{d \mu(x)}{z-x}, \quad z \in \mathbf{C} \backslash B .
$$

Let $\left\{b_{n}\right\}, n \in \mathbf{N}$, be a sequence of monic polynomials whose zeros lie in $B$ and has constant sign on that interval. Assume that $\operatorname{deg} B_{n}=m(n) \leq n$. Therefore,

$$
B_{n}(z)=\prod_{k=1}^{m(n)}\left(z-\beta_{n, k}\right), \quad \beta_{n, k} \in B .
$$

Let us fi a compact set $A \subset \overline{\mathbf{C}} \backslash B$ that is symmetric with respect to the real line and a table of points

$$
\left\{\alpha_{n, i}\right\}, \quad i=1,2, \ldots, 2 n-m(n), \quad n \in \mathbf{N},
$$

contained in $A$ that is also symmetric with respect to the real line (counting multiplicities). Set

$$
A_{n}(z)=\prod_{i=1}^{2 n-m(n)}\left(z-\alpha_{n, i}\right), \quad n \in \mathbf{N} .
$$

In the case that for some $i, \alpha_{n, i}=\infty$, we omit the corresponding factor. By symmetry all the coefficient of $A_{n}$ are real and since $A \cap B=\emptyset, A_{n}$ has constant sign on $B$. It is easy to verify that for each $n \in \mathbf{N}$, there exists a unique rational function of the form

AMS classification: Primary 41A21, 41A25; Secondary 30E10, $42 \mathrm{C} 05$.

Key words and phrases: Padé-type approximation, Multipoint approximation, Rate of Convergence, Quadratures. 
$R_{n}=P_{n} / B_{n} Q_{n}$, where $P_{n}$ and $Q_{n}$ are polynomials satisfying:

(a) $\operatorname{deg} P_{n} \leq n-1 ; \operatorname{deg} Q_{n} \leq n-m(n)$; and $Q_{n} \not \equiv 0$.

(b) $\frac{Q_{n} B_{n} \hat{\mu}-P_{n}}{A_{n}} \in H(\mathbf{C} \backslash B)$.

(c) $\frac{Q_{n} B_{n} \hat{\mu}-P_{n}}{A_{n}}(z)=O\left[\frac{1}{z^{n-m(n)+1}}\right]$ as $z \rightarrow \infty$.

The determination of $P_{n}$ and $Q_{n}$ may be reduced to the solution of a homogeneous system of $2 n-m(n)$ equations in $2 n-m(n)+1$ unknowns, thus a nontrivial solution $\left(Q_{n} \not \equiv 0\right)$ always exists. We will take $Q_{n}$ monic.

When, for all $n \in \mathbf{N}, m(n)=0$, all the poles of $R_{n}$ are free and this construction coincides with that of the diagonal multipoint Padé approximant which interpolates $\hat{\mu}$ at the zeros of $A_{n}$. In this case, the convergence of the corresponding sequence of rational functions $\left\{R_{n}\right\}$ has been well studied and estimates of the rate of convergence have been given (see, e.g., [3] and [8]). When $m(n)=n$ all the poles are fi ed and the study of the convergence of such approximants parallels in great degree the theory of approximation of analytic functions by means of polynomials (this situation is extensively treated in [9]). We will discuss the intermediate cases when $m(n)$ poles are fi ed and $n-m(n)$ are left free.

The object of this paper is to fin conditions on $\left(\mu,\left\{A_{n}\right\},\left\{B_{n}\right\},\{m(n)\}\right)$ so that there exists

$$
\lim _{n}\left\|\hat{\mu}-R_{n}\right\|_{K}^{1 / n}
$$

where $\|\cdot\|_{K}$ denotes the sup-norm on a compact set $K$ contained in $\overline{\mathbf{C}} \backslash(A \cup B)$ and determine the limit value (see Theorem 1 below).

In the classical setting, when interpolation is carried out at infinit $\left(A_{n} \equiv 1, n \in \mathbf{N}\right)$, a firs paper on this subject was provided by [5]. The authors consider the situation when $\lim _{n} m(n) / n=0$; that is, nearly Padé approximation. Later, in the same framework, we studied the problem when $\lim _{n} m(n) / n=\theta \in[0,1]$ (see [2]). Recently, we received a preprint of A. Ambroladze and H. Wallin [1] where they consider, also in the classical setting, the problem of upper estimates for

$$
\varlimsup_{n}\left\|\hat{\mu}-R_{n}\right\|_{K}^{1 / n} .
$$

They also considerably weaken the condition $\mu^{\prime}>0$ a.e. that we required on $\mu$ in [2] in order that (1) takes place.

A key question in the solution of the problems above is the study of equilibrium distributions in the presence of exterior fields Our paper is particularly influence by the results contained in [4] which are ready to use for our purpose. We also refer the reader to the excellent monograph [8] whose methods may also be applied to obtain similar results to the ones we give here under weaker assumptions (this is the main strategy of [1] in contrast with [2]). The results in [8 (see Section 3.3)] must be adapted in order that they cover the type of varying weights we consider here. Therefore, we restrict generality in favor of simplicity. Nevertheless, our restrictions are sufficientl general to include the most interesting applications (see the corollaries below). In the following, we maintain the notations introduced above and the conditions on $\left(\mu,\left\{A_{n}\right\},\left\{B_{n}\right\},\{m(n)\}\right)$. 


\section{Some Lemmas}

First let us show that the polynomials $Q_{n}$ satisfy certain orthogonality relations with respect to a varying measure and fin an integral expression for the error in the approximation.

Lemma 1. We have

$$
\int x^{j} Q_{n}(x) \frac{B_{n}(x) d \mu(x)}{A_{n}(x)}=0, \quad j=0,1, \ldots, n-m(n)-1,
$$

and

$$
\left(\hat{\mu}-R_{n}\right)(z)=\frac{A_{n}(z)}{\left(B_{n} Q_{n} h\right)(z)} \int \frac{\left(B_{n} Q_{n} h\right)(x)}{A_{n}(x)} \frac{d \mu(x)}{z-x}, \quad z \in \overline{\mathbf{C}} \backslash B,
$$

where $h$ is any polynomial of degree $\leq n-m(n)$.

Proof. Multiply (c) times $z^{j}, j=0,1, \ldots, n-m(n)-1$, and integrate along any contour $\Gamma$ which surrounds $B(B \subset \operatorname{Int}(\Gamma))$ in such a way that $A$ lies outside the closed domain determined by $\Gamma(A \subset \operatorname{Ext}(\Gamma))$. From Cauchy's theorem, Fubini's theorem, and Cauchy's integral formula, it follows that

$$
\begin{aligned}
0 & =\int_{\Gamma} z^{j} \frac{Q_{n} B_{n} \hat{\mu}-P_{n}}{A_{n}}(z) d z=\int_{\Gamma} z^{j} \frac{Q_{n} B_{n} \hat{\mu}}{A_{n}}(z) d z \\
& =\iint_{\Gamma} z^{j} \frac{Q_{n} B_{n}}{A_{n}}(z) \frac{d z}{z-x} d \mu(z)=2 \pi i \int x^{j} \frac{Q_{n} B_{n}}{A_{n}}(x) d \mu(x) .
\end{aligned}
$$

This proves (2).

On the other hand, if $z \in \operatorname{Ext}(\Gamma)$ and $h$ is an arbitrary polynomial of degree $\leq n-m(n)$, using Cauchy's integral formula and Fubini's theorem we obtain

$$
\begin{aligned}
h(z) \frac{Q_{n} B_{n} \hat{\mu}-P_{n}}{A_{n}}(z) & =\frac{1}{2 \pi i} \int_{\Gamma} h(\zeta) \frac{Q_{n} B_{n} \hat{\mu}-P_{n}}{A_{n}}(\zeta) \frac{d \zeta}{z-\zeta} \\
& =\frac{1}{2 \pi i} \int_{\Gamma} \frac{\left(h Q_{n} B_{n} \hat{\mu}\right)(\zeta)}{A_{n}(\zeta)} \frac{d \zeta}{z-\zeta} \\
& =\int \frac{1}{2 \pi i} \int_{\Gamma} \frac{\left(h Q_{n} B_{n}\right)(\zeta)}{A_{n}(\zeta)(z-\zeta)} \frac{d \zeta}{\zeta-x} d \mu(x) \\
& =\int \frac{\left(h Q_{n} B_{n}\right)(x)}{A_{n}(x)(z-x)} d \mu(x) .
\end{aligned}
$$

Therefore, (3) is proved.

Denote

$$
N_{n}=\left(\int\left|\frac{\left(Q^{2} B_{n}\right)(x)}{A_{n}(x)}\right| d \mu(x)\right)^{1 / 2}, \quad q_{n}=\frac{Q_{n}}{N_{n}} .
$$

Taking $h=Q_{n} / N_{n}^{2}$ in (3), we obtain

$$
\left(\hat{\mu}-R_{n}\right)(z)=\frac{A_{n}(z)}{\left(B_{n} q_{n}^{2}\right)(z)} \int \frac{\left(B_{n} q_{n}^{2}\right)(x)}{A_{n}(x)} \frac{d \mu(x)}{z-x}, \quad z \in \mathbf{C} \backslash B .
$$


From (4) it is easy to check the following bounds for the error formula (see [8, $(6.1 .34)])$ :

Lemma 2. There exist two positive continuous functions $d_{1}(z)>0$ and $d_{2}(z)<\infty$ on $\overline{\mathbf{C}} \backslash$ B such that

$$
d_{1}(z)\left|\frac{A_{n}(z)}{\left(B_{n} q_{n}^{2}\right)(z)}\right| \leq\left|\left(\hat{\mu}-R_{n}\right)(z)\right| \leq d_{2}(z)\left|\frac{a_{n}(z)}{\left(B_{n} q_{n}^{2}\right)(z)}\right| .
$$

Since $d_{1}(z)$ and $d_{2}(z)$ are bounded from below and from above on each compact set contained in $\mathbf{C} \backslash B$, formula (5) guarantees that the $n$th root asymptotic behavior of the error is completely determined by that of the sequence $\left\{\left|A_{n}(z) /\left(B_{n} q_{n}^{2}\right)(z)\right|\right\}$.

Notice (see (2)) that $q_{n}$ is the polynomial of degree $n-m(n)$ orthonormal with respect to the varying measure

$$
d \mu(x)=\frac{B_{n}(x) d \mu(x)}{A_{n}(x)} .
$$

The $n$th root asymptotic behavior of such polynomials has been well studied (see [4] and [8]). It depends on the asymptotic behavior of the functions $\left(\left|B_{n}(x) / A_{n}(x)\right|\right)^{1 / n}$ as $n \rightarrow \infty$ and the limit is described in terms of the solution of an equilibrium problem in the presence of an exterior field For convenience in the reading, we give the corresponding result as presented in [4] under the restrictions with which we are working.

Without loss of generality we will assume in the following that $A$ is compactly contained in $\mathbf{C} \backslash B$ (see, e.g., Section 6.1 in [8]). This assumption simplifie the consideration of the measures and their corresponding potentials and any problem of type (1) may be reduced to this situation by means of a simple Möbius transformation. In particular, in the following, $\operatorname{deg} A_{n}=2 n-m(n)$. Let $\alpha_{n}$ denote the counting measure for the zeros of $A_{n}$ and $\beta_{n}$ the counting measure for the zeros of $B_{n}$. For any measure $\sigma$, we denote its logarithmic potential by

$$
V_{\sigma}(z)=\int \log |z-t|^{-1} d \sigma(t)
$$

Set

$$
f_{n}(x)=\frac{1}{2(n-m(n))}\left[V_{\beta_{n}}(x)-V_{\alpha_{n}}(x)\right] .
$$

Assume that the following limit exists

$$
f(x)=\lim _{n} f_{n}(x), \quad x \in B
$$

where $f$ is not identically equal to $+\infty$ on $B$. From the known results of potentials it immediately follows that the following properties take place (see, e.g., [4] and [6]):

(a') Each $f_{n}$ as well as $f$ is lower semicontinuous on $B$.

(b') Each $f_{n}$ as well as $f$ is weakly approximatively continuous on $B$. A function $g$ is weakly approximatively continuous at a point $x_{0} \in B$, if there exists a set $e\left(x_{0}\right)$ 
(depending on $g$ ) of positive density at $x_{0}$ such that

$$
\varliminf_{\substack{x \rightarrow x_{0} \\ x \in B}} g(x)=\lim _{\substack{x \rightarrow x_{0} \\ x \in e\left(x_{0}\right)}} g(x)=g\left(x_{0}\right) .
$$

(c') $\lim _{n \rightarrow \infty} \min _{B} f_{n}(x)=\min _{B} f(x)$.

Due to $\left(\mathrm{a}^{\prime}\right)-\left(\mathrm{c}^{\prime}\right)$, from Theorem 1 in [4], we have:

Lemma 3. If $\mu^{\prime}>0$ a.e. on $B$ and (6) takes place, then

$$
\lim _{n} N_{n}^{1 /(n-m(n))}=\exp \{-\omega\},
$$

and

$$
\lim _{n}\left|Q_{n}(z)\right|^{1 /(n-m(n))}=\exp \left\{-V_{\lambda}(z)\right\}
$$

uniformly on each subset of $\mathbf{C} \backslash B$, where $\omega$ is the extremal constant and $\lambda$ the unit equilibrium measure on $B$ which are uniquely determined and characterized by the relations

$$
\begin{aligned}
V_{\lambda}(x)+f(x) & =\omega, & & x \in \operatorname{supp}(\lambda), \\
& \geq \omega, & x & \in B .
\end{aligned}
$$

Lemmas 2 and 3 allow us to obtain a result of type (1) in terms of $f, \lambda$, and $\omega$. Unfortunately, under such general conditions, it is not possible to fin explicitly the extremal constant and the equilibrium measure. Therefore, we will restrict our attention to a sufficientl general case which contains the more interesting applications.

Assume that

$$
\lim _{n} \frac{m(n)}{n}=\theta \in[0,1] .
$$

We will also assume the existence of unit measures $\alpha$ and $\beta(\operatorname{supp}(\alpha) \subset A$, $\operatorname{supp}(\beta) \subset B)$, such that

$$
\lim _{n} \frac{1}{n} \alpha_{n}=(2-\theta) \alpha, \quad \lim \frac{1}{n} \beta_{n}=\theta \beta,
$$

where $\lim _{n}$ in connection with measures stands for convergence in the weak-star topology. Notice that (11) (on account of (10)) simply means that the corresponding sequence of measures have limit. For further reference, we indicated the form that such limit measures must have.

Let use denote by $\omega^{*}$ and $\lambda^{*}$ the extremal constant and the equilibrium measure which solve the extremal problem (9) when $f(x)=-V_{\alpha}(x) . \lambda^{*}$ is the balayage on $B$ of $\alpha$ and it is well known that $\operatorname{supp}\left(\lambda^{*}\right)=B$ (see [6]). We have the explicit expressions (see [4] and [7])

$$
\omega^{*}=\int g_{B}(t, \infty) d \alpha(t)
$$

and

$$
d \lambda^{*}(x)=\frac{d x}{\pi\left(1-x^{2}\right)^{1 / 2}} \int \frac{\left(t^{2}-1\right)^{1 / 2}}{|x-t|} d \alpha(t),
$$


where $g_{B}(t, \infty)$ denotes Green's function for the region $\overline{\mathbf{C}} \backslash B$ with singularity at $\infty$.

Finally, we will assume that the measures $\alpha$ and $\beta$ are such that

$$
(2-\theta) \lambda^{*}-\theta \beta \geq 0 .
$$

By this we indicate that such a difference is a positive measure. This last condition is rather restrictive if $\theta=1$, since then $\beta$ is forced to be equal to $\lambda^{*}$, but we are still free to select any sequence of polynomials $\left\{A_{n}\right\}$ which have $n$th root asymptotics.

Lemma 4. Let $\mu^{\prime}>0$ a.e. on B. Assume that (10), (11), and (14) take place. Then,

$$
\lim _{n} N_{n}^{1 / n}=\exp \left\{-\frac{2-\theta}{2} \omega^{*}\right\},
$$

and

$$
\lim _{n}\left|Q_{n}(z)\right|^{1 / n}=\exp \left\{-\frac{1}{2} V_{(2-\theta) \lambda^{*}-\theta \beta}(z)\right\},
$$

uniformly on each compact subset of $\mathbf{C} \backslash B$, where $\omega^{*}$ and $\lambda^{*}$ are as indicated above (and equal to (12) and (13) when $A$ is a segment contained in $\mathbf{R} \backslash B$ ).

Proof. First let us consider that $\theta \in[0,1)$. In this case, we have

$$
f(x)=\frac{1}{2(1-\theta)}\left[\theta V_{\beta}(x)-(2-\theta) V_{\alpha}(x)\right] .
$$

Thus (6) takes place. According to (7) and (8)

$$
\lim _{n} N_{n}^{1 / n}=\exp \{-(1-\theta) \omega\},
$$

and

$$
\lim _{n}\left|Q_{n}(z)\right|^{1 / n}=\exp \left\{-(1-\theta) V_{\lambda}(z)\right\},
$$

where $\omega$ and $\lambda$ solve the extremal problem on $B$ in the presence of the exterior fiel given by (17). That is,

$$
\begin{array}{rlrl}
V_{\lambda}(x)+\frac{\theta}{2(1-\theta)} V_{\beta}(x)-\frac{2-\theta}{2(1-\theta)} V_{\alpha}(x) & =\omega, & & x \in \operatorname{supp}(\lambda), \\
& \geq \omega, \quad & x \in B .
\end{array}
$$

Notice that $\lambda+[\theta / 2(1-\theta)] \beta$ and $[(2-\theta) / 2(1-\theta)] \alpha$ are measures on $B$ and $A$, respectively, each of which has total mass $(2-\theta) / 2(1-\theta)$. Therefore, from the uniqueness of the solution of this extremal problem and that when $f(x)=-V_{\alpha}(x)$, it follows that if (14) takes place (notice that $\operatorname{supp}\left(\lambda^{*}\right)=B \supset \operatorname{supp}(\lambda)$ ), then

$$
\omega=\frac{2-\theta}{2(1-\theta)} \omega^{*}
$$

and

$$
\lambda=\frac{1}{2(1-\theta)}\left[(2-\theta) \lambda^{*}-\theta \beta\right] .
$$

Substituting these formulas into (18) and (19), we obtain (15) and (16), respectively. 
Let us consider $\theta=1$. Now condition (14) requires that $\beta=\lambda^{*}$. Thus (16) reduces to proving that

$$
\lim _{n}\left|Q_{n}(z)\right|^{1 / n}=1 .
$$

But this is obvious because on each compact subset $K$ of $\mathbf{C} \backslash B$

$$
(\mathrm{d}(K, B))^{n-m(n)} \leq\left\|Q_{n}(z)\right\|_{K} \leq(\operatorname{diam}(K \cup B))^{n-m(n)},
$$

where $\mathrm{d}(K, B)$ denotes the distance between the nonintersecting compact sets $K$ and $B$, while diam $(K \cup B)$ denotes the diameter of their union.

In connection with (15), from the definitio it immediately follows that

Let $\varepsilon>0$. Set

$$
N_{n} \leq 2^{n-m(n)}\left\|\frac{B_{n}}{A_{n}}\right\|_{B}^{1 / 2}|\mu|^{1 / 2} .
$$

$$
\Gamma_{\varepsilon}=\left\{z \in \mathbf{C}: V_{\lambda^{*}}(z)-V_{\alpha}(z)=\omega^{*}-\varepsilon\right\} .
$$

Take $\varepsilon$ sufficientl small so that $\Gamma_{\varepsilon}$ surrounds $B$ and $A \subset \operatorname{Ext}\left(\Gamma_{\varepsilon}\right)$. From the maximum principle, (10) and (11), we obtain

$$
\varlimsup_{n} N_{n}^{1 / n} \leq \lim _{n}\left\|\frac{B_{n}}{A_{n}}\right\|_{\Gamma_{\varepsilon}}^{1 / 2 n}=\exp \left\{-\frac{1}{2} \omega^{*}\right\} .
$$

Taking $\varepsilon \rightarrow 0$, we obtain

$$
\varlimsup_{n} N_{n}^{1 / n} \leq \exp \left\{-\frac{1}{2} \omega^{*}\right\}
$$

On the other hand, $B_{n}(x)$ has constant sign on $B$; therefore,

$$
B_{n}(x)=\left(B_{n}^{*}(x)\right)^{2} t_{n}(x),
$$

where $t_{n}$ is a polynomial of degree at most two, whose zeros should they exist must lie at the endpoints of $B$. Thus $B_{n}^{*} Q_{n}$ is a monic polynomial such that

$$
n-\frac{m(n)}{2}-1 \leq m^{*}(n)=\operatorname{deg}\left(B_{n}^{*} Q_{n}\right) \leq n-\frac{m(n)}{2} .
$$

Denote by $L_{n}$ the monic polynomial of degree $m^{*}(n)$ orthogonal with respect to the measure $\left|t_{n}(x) / A_{n}(x)\right| d \mu(x)$. This polynomial is of minimal $L_{2}$-norm with respect to this measure between all monic polynomials of degree $m^{*}(n)$. Therefore,

$$
\begin{aligned}
N_{n}^{*} & =\left\{\int\left|L_{n}(x)\right|^{2}\left|\frac{t_{n}(x)}{A_{n}(x)}\right| d \mu(x)\right\}^{1 / 2} \\
& \leq\left\{\int\left|\left(B_{n}^{*} Q_{n}\right)(x)\right|^{2}\left|\frac{t_{n}(x)}{A_{n}(x)}\right| d \mu(x)\right\}^{1 / 2}=N_{n} .
\end{aligned}
$$

Since $\left|t_{n}(x)\right| d \mu(x)>0$ a.e. on $B$, from Lemma 3 as applied to the sequence of polynomials $\left\{L_{n}\right\}$ (see also Theorem 1 in [4]), we obtain

$$
\lim _{n}\left(N_{n}^{*}\right)^{1 / m^{*}(n)}=\exp \left\{-\frac{1}{2} \omega^{*}\right\}
$$

From (21)-(23), it follows that

$$
\frac{\lim _{n}}{n} N_{n}^{1 / n} \geq \exp \left\{-\frac{1}{2} \omega^{*}\right\}
$$

This inequality together with (20) give (15) for $\theta=1$. With this we conclude the proof. 


\section{Main Results}

We are ready for the proof of

Theorem 1. Let $\mu^{\prime}>0$ a.e. on B. Assume that (10), (11), and (14) take place. Then, on each compact subset $K \subset \mathbf{C} \backslash(A \cup B)$, we have

$$
\lim _{n}\left\|\hat{\mu}-R_{n}\right\|_{K}^{1 / n}=\sup _{z \in K} \exp \left\{(\theta-2)\left[\omega^{*}-V_{\lambda^{*}-\alpha}(z)\right]\right\} .
$$

Moreover, on each compact subset $K \subset \mathbf{C} \backslash B$ such that $\operatorname{cap}(K)>0$,

$$
\varlimsup_{n}\left\|\hat{\mu}-R_{n}\right\|_{K}^{1 / n}=\sup _{z \in K} \exp \left\{(\theta-2)\left[\omega^{*}-V_{\lambda^{*}-\alpha}(z)\right]\right\},
$$

where $\operatorname{cap}(\cdot)$ denotes the logarithmic capacity of the indicated set.

Proof. Take $K$ as indicated above depending on whether it is contained in $\mathbf{C} \backslash(A \cup B)$ or $\mathbf{C} \backslash B$. According to (5), on account of the properties which $d_{1}(z)$ and $d_{2}(z)$ satisfy on $\mathbf{C} \backslash B$, we have

$$
\lim _{n}\left\|\hat{\mu}-R_{n}\right\|_{K}^{1 / n}=\lim _{n}\left\|\frac{A_{n}}{B_{n} q_{n}^{2}}\right\|_{K}^{1 / n},
$$

for $K \subset \mathbf{C} \backslash(A \cup B)$, and

$$
\varlimsup_{n}\left\|\hat{\mu}-R_{n}\right\|_{K}^{1 / n}=\varlimsup_{n}\left\|\frac{A_{n}}{B_{n} q_{n}^{2}}\right\|_{K}^{1 / n},
$$

for $K \subset \mathbf{C} \backslash B$, should the limits on the right-hand side of (26) and (27) exist.

Each factor under the norm sign on the right-hand sides have $n$th root asymptotics. From (11) and the Lower Envelope Theorem (see [6, Theorem 3.8])

$$
\varlimsup_{n}\left|A_{n}(z)\right|^{1 / n}=\exp \left\{(\theta-2) V_{\alpha}(z)\right\},
$$

uniformly on each compact subset of positive capacity of $\mathbf{C}$, with the existence of limit in case that the compact set is contained in $\mathbf{C} \backslash A$ without the requirement that it have positive capacity. From (11), (15), and (16)

$$
\lim _{n}\left|\left(B_{n} q_{n}^{2}\right)(z)\right|^{1 / n}=\exp \left\{(\theta-2)\left[\omega^{*}-V_{\lambda^{*}}(z)\right]\right\},
$$

uniformly on each compact subset of $\mathbf{C} \backslash B$.

Notice that the right-hand side of (28) is bounded away from $+\infty$ on each compact subset of $\mathbf{C}$, while the right-hand side of (29) is bounded away from zero on each compact subset of $\mathbf{C} \backslash B$. From (26)-(29), readily follow (24) and (25).

Remark 1. We have $\operatorname{supp}\left(\lambda^{*}\right)=B$; therefore, from (9) we know that $V_{\lambda^{*}-\alpha}(z) \equiv \omega^{*}$ on $B$. Since $V_{\lambda^{*}-\alpha}(z)$ is subharmonic on $\overline{\mathbf{C}} \backslash B$, from the maximum principle, we have that $V_{\lambda^{*}-\alpha}(z)<\omega^{*}$ on $\overline{\mathbf{C}} \backslash B$ (equality is not possible at any point of $\overline{\mathbf{C}} \backslash B$ since $V_{\lambda^{*}-\alpha}(z)$ cannot be constantly equal to $\omega^{*}$ on all $\left.\overline{\mathbf{C}}\right)$. Therefore, (24) and (25) indicate that the Padé-type approximants converge uniformly to $\hat{\mu}$ with geometric rate on each compact subset of $\mathbf{C} \backslash(A \cup B)$ and the exact rate is attained. 
Corollary 1. Let $\mu^{\prime}>0$ a.e. on B. Assume that (10), (11), and (14) take place with $\lim _{n \in \Lambda}$ in place of $\lim _{n}$, where $\Lambda \subset \mathbf{N}$ is an infinite sequence of indexes. Then, on each compact subset $K \subset \mathbf{C} \backslash(A \cup B)$, we have

$$
\lim _{n \in \Lambda}\left\|\hat{\mu}-R_{n}\right\|_{K}^{1 / n}=\sup _{z \in K} \exp \left\{(\theta-2)\left[\omega^{*}-V_{\lambda^{*}-\alpha}(z)\right]\right\} .
$$

On each compact subset $K \subset \mathbf{C} \backslash B$ such that cap $(K)>0$,

$$
\varlimsup_{n \in \Lambda}\left\|\hat{\mu}-R_{n}\right\|_{K}^{1 / n}=\sup _{z \in K} \exp \left\{(\theta-2)\left[\omega^{*}-V_{\lambda^{*}-\alpha}(z)\right]\right\} .
$$

Proof. It is an immediate consequence of the fact that in the proof of Theorem 1 no use is made of the fact that the limits are taken following the complete sequence of natural numbers.

Let $\Lambda \subset \mathbf{N}$ be an infinit sequence of indexes and $\tau$ and a measure. We say that

$$
\varlimsup_{n \in \Lambda}(1 / n) \beta_{n} \leq \tau
$$

if any convergent subsequence $\left\{(1 / n) \beta_{n}\right\}, n \in \Lambda^{\prime} \subset \Lambda$, satisfie that $\tau-\lim _{n \in \Lambda^{\prime}}(1 / n) \beta_{n} \geq$ 0 (is a positive measure on $B$ ). In all that follows

$$
\theta_{1}=\frac{\lim }{n} \frac{m(n)}{n} \quad \text { and } \quad \theta_{2}=\varlimsup_{n} \frac{m(n)}{n} .
$$

From Corollary 1 the following generalization of Theorem 1 is easy to obtain.

Corollary 2. Let $\mu^{\prime}>0$ a.e. on B. Assume that there exists a fixed unit positive Borel measure $\alpha$ such that for each sequence of indexes $\Lambda \subset \mathbf{N}$ for which $\left\{(1 / n) \alpha_{n}\right\}, n \in \Lambda$, is convergent, there exists $\theta_{\Lambda}$ such that

$$
\lim _{n \in \Lambda} \frac{1}{n} \alpha_{n}=\left(2-\theta_{\Lambda}\right) \alpha \quad \text { and } \quad \varlimsup_{n \in \Lambda} \frac{1}{n} \beta_{n} \leq\left(2-\theta_{\Lambda}\right) \lambda^{*} .
$$

Then, on each compact subset $K \subset \mathbf{C} \backslash(A \cup B)$, we have

$$
\underline{\lim _{n}}\left\|\hat{\mu}-R_{n}\right\|_{K}^{1 / n}=\sup _{z \in K} \exp \left\{\left(\theta_{1}-2\right)\left[\omega^{*}-V_{\lambda^{*}-\alpha}(z)\right]\right\},
$$

and

$$
\varlimsup_{n}\left\|\hat{\mu}-R_{n}\right\|_{K}^{1 / n}=\sup _{z \in K} \exp \left\{\left(\theta_{2}-2\right)\left[\omega^{*}-V_{\lambda^{*} \alpha}(z)\right]\right\} .
$$

On each compact subset $K \subset \mathbf{C} \backslash B$ such that $\operatorname{cap}(K)>0$,

$$
\varlimsup_{n}\left\|\hat{\mu}-R_{n}\right\|_{K}^{1 / n}=\sup _{z \in K} \exp \left\{\left(\theta_{2}-2\right)\left[\omega^{*}-V_{\lambda^{*}-\alpha}(z)\right]\right\} .
$$

Additionally, if (10) takes place, then (24) and (25) hold true.

Proof. Let $\theta$ be any one of the accumulation points of the sequence $\{m(n) / n\}, n \in \mathbf{N}$, then there exists a sequence of indexes $\Lambda \subset \mathbf{N}$ such that

$$
\lim _{n \in \Lambda} \frac{m(n)}{n}=\theta=\theta_{\Lambda} \quad\left(\in\left[\theta_{1}, \theta_{2}\right]\right) .
$$


The family of measures $\left\{(1 / n) \alpha_{n}\right\},\left\{(1 / n) \beta_{n}\right\}$, are relatively compact in the weak-star topology; therefore, using (32), there exists a subsequence of indexes $\Lambda^{\prime} \subset \Lambda$, such that

$$
\lim _{n \in \Lambda^{\prime}} \frac{1}{n} \alpha_{n}=\left(2-\theta_{\Lambda}\right) \alpha, \quad \lim _{n \in \Lambda^{\prime}} \frac{1}{n} \beta_{n}=\theta_{\Lambda} \beta_{\Lambda}, \quad\left(2-\theta_{\Lambda}\right) \lambda^{*}-\theta_{\Lambda} \beta_{\Lambda} \geq 0 .
$$

From Corollary 1, we conclude that:

(a) On each compact subset $K \subset \mathbf{C} \backslash(A \cup B)$, we have

$$
\lim _{n \in \Lambda^{\prime}}\left\|\hat{\mu}-R_{n}\right\|_{K}^{1 / n}=\sup _{z \in K} \exp \left\{\left(\theta_{\Lambda}-2\right)\left[\omega^{*}-V_{\lambda^{*}-\alpha}(z)\right]\right\} .
$$

(b) On each compact subset $K \subset \mathbf{C} \backslash B$ such that $\operatorname{cap} K>0$,

$$
\varlimsup_{n \in \Lambda^{\prime}}\left\|\hat{\mu}-R_{n}\right\|_{K}^{1 / n}=\sup _{z \in K} \exp \left\{\left(\theta_{\Lambda}-2\right)\left[\omega^{*}-V_{\lambda^{*}-\alpha}(z)\right]\right\} /
$$

The sequence $\{m(n) / n\}, n \in \mathbf{N}$, is also relatively compact in the usual topology of $\mathbf{R}$, therefore the limsup and liminf in (33)-(35) must be equal to some expression as in the right-hand side of (36) and (37) where $\theta_{\Lambda}$ is one of the accumulation points of the sequence $\{m(n) / n\}, n \in \mathbf{N}$. Since the factor $\left[\omega^{*}-V_{\lambda^{*}-\alpha}(z)\right]$ does not depend on $\Lambda$, (33)-(35) follow. The last statement in Corollary 2 is an immediate consequence of (33)-(35).

The next two corollaries are particular cases of special interest.

Assume that $A$ reduces to a single point $a$ of the real line. Denote by $\delta_{a}$ the Dirac measure with mass at $\{a\}$. $\delta_{a}$ will play the role of $\alpha$ in Corollary 2. Obviously, $\left\{(1 / n) \alpha_{n}\right\}$, $n \in \Lambda$, is convergent, if and only if there exists $\theta_{\Lambda}$ such that

$$
\lim _{n \in \Lambda} \frac{1}{n} \alpha_{n}=\left(2-\theta_{\Lambda}\right) \delta_{a}
$$

In this case, $-V_{\alpha}(z)=-V_{\delta_{a}}(z)=-\log |z-a|^{-1}$, and using (12)-(13), we can fin the corresponding $\omega^{*}$ and $\lambda^{*}$. In fact,

$$
\omega^{*}=g_{B}(a, \infty) \quad \text { and } \quad d \lambda^{*}(x)=\frac{\left(a^{2}-1\right)^{1 / 2}}{|x-a|} \frac{d x}{\pi\left(1-x^{2}\right)^{1 / 2}} .
$$

But in this simple case, in order to obtain a reduced expression of the right-hand sides in (33)-(35) it is better to proceed differently. Equation (9) indicates that

$$
\omega^{*}-V_{\lambda^{*}}(x)+\log |x-a|^{-1} \equiv 0, \quad x \in B .
$$

Since $\omega^{*}-V_{\lambda^{*}}(z)+\log |z-a|^{-1}$ is harmonic in $\overline{\mathbf{C}} \backslash(B \cup\{a\})$ and behaves like log $|z-a|^{-1}$ on a neighborhood of $\{a\}$, it follows that

$$
\omega^{*}-V_{\lambda^{*}-\alpha}(z)=\omega^{*}-V_{\lambda^{*}}(z)+\log |a-z|^{-1} \equiv g_{B}(z, a),
$$

where $g_{B}(z, a)$ denotes Green's function for the region $\overline{\mathbf{C}} \backslash B$ with singularity at point $\{a\}$. It is well known and easy to verify that

$$
g_{B}(z, a)=-\log |\varphi(z, a)|,
$$

where $\varphi(z, a)$ is any conformal representation of $\overline{\mathbf{C}} \backslash B$ onto the interior of the unit disk which transforms point $a$ into 0 .

In the following corollary $\theta_{\Lambda}$ is as indicated in (38) and $\lambda^{*}$ as in (39). 
Corollary 3. Let $\mu^{\prime}>0$ a.e. on $B$ and $A=\{a\}$. Assume that

$$
\varlimsup_{n \in \Lambda} \frac{1}{n} \beta_{n} \leq\left(2-\theta_{\Lambda}\right) \lambda^{*} .
$$

Then, on each subset $K \subset \backslash(\mathbf{A} \cup \mathbf{B})$, we have

$$
\frac{\lim }{n}\left\|\hat{\mu}-R_{n}\right\|_{K}^{1 / n}=\sup _{z \in K}|\varphi(z, a)|^{2-\theta_{1}},
$$

and

$$
\varlimsup_{n}\left\|\hat{\mu}-R_{n}\right\|_{K}^{1 / n}=\sum_{z \in K}|\varphi(z, a)|^{2-\theta_{2}} .
$$

On each compact subset $K \subset \mathbf{C} \backslash B$ such that $\operatorname{cap}(K)>0$,

$$
\varlimsup_{n}\left\|\hat{\mu}-R_{n}\right\|_{K}^{1 / n}=\sup _{z \in K}|\varphi(z, a)|^{2-\theta_{2}} .
$$

Remark 2. Corollary 3 reduces to the theorem and corollary in [2] when $a=\infty$. In this case, condition (40) is replaced by

$$
\varlimsup_{n \in \Lambda} \frac{1}{n} \beta_{n} \leq\left(2-\theta_{\Lambda}\right) \frac{d x}{\pi\left(1-x^{2}\right)^{1 / 2}},
$$

where $\theta_{\Lambda}=\lim _{n \in \Lambda} m(n) / n$ is any one of the accumulation points of the sequence $\{m(n) / n\}, n \in \mathbf{N}$, and

$$
|\varphi(z, \infty)|=\left|z-\left(z^{2}-1\right)^{1 / 2}\right|
$$

In the next application $A$ is a compact set with connected complement which is regular with respect to Dirichlet's problem. Let $h(z)$ be the harmonic function in $\overline{\mathbf{C}} \backslash(A \cup B)$ which can be continuously extended to all $\overline{\mathbf{C}}$ with constant value 0 on $B$ and 1 on $A$. Let $C=C(A, B)$ be the capacity of the condenser $(A, B)$ (also called Green's capacity of $A$ in the region $\overline{\mathbf{C}} \backslash B$; see, e.g., [6, Chap. II, No. 18], also [3]). Denote $H(z)=(1 / C)(h(\infty)-h(z))$.

From Green's formula it is possible to obtain the following representation:

$$
H(z)=V_{\lambda_{B}} \quad \text { and } \quad \omega^{*}=\frac{1}{C} h(\infty),
$$

when we take on $B$ the exterior fiel induced by $\alpha=\lambda_{A}$.

Corollary 4. Let $\mu^{\prime}>0$ a.e. on $B$. Assume that for each sequence of indexes $\Lambda \subset \mathbf{N}$ for which $\left\{(1 / n) \alpha_{n}\right\}, n \in \Lambda$, is convergent, there exists $\theta_{\Lambda}$ such that

$$
\lim _{n \in \Lambda} \frac{1}{n} \alpha_{n}=\left(2-\theta_{\Lambda}\right) \lambda_{A} \quad \text { and } \quad \varlimsup_{n \in \Lambda} \frac{1}{n} \beta_{n} \leq\left(2-\theta_{\Lambda}\right) \lambda_{B} .
$$


Then, on each compact subset $K \subset \mathbf{C} \backslash(A \cup B)$, we have

$$
\frac{\lim }{n}\left\|\hat{\mu}-R_{n}\right\|_{K}^{1 / n}=\sup _{z \in K} \exp \left\{\frac{\left.\theta_{1}-2\right) h(z)}{C}\right\},
$$

and

$$
\varlimsup_{n}\left\|\hat{\mu}-R_{n}\right\|_{K}^{1 / n}=\sup _{z \in K} \exp \left\{\frac{\left(\theta_{2}-2\right) h(z)}{C}\right\} .
$$

On each compact subset $K \subset \mathbf{C} \backslash B$ such that $\operatorname{cap}(K)>0$,

$$
\varlimsup_{n}\left\|\hat{\mu}-R_{n}\right\|_{K}^{1 / n}=\sup _{z \in K} \exp \left\{\frac{\left(\theta_{2}-2\right) h(z)}{C}\right\} .
$$

Remark 3. A version of Corollary 4 for multipoint Padé approximants is Theorem 2 in [3] (we also refer to Theorem 6.1.6 in [8]).

Remark 4. The restriction $\mu^{\prime}>0$ a.e. comes exclusively from Lemma 3 which as pointed out above is an immediate consequence of Theorem 1 in [4]. Following their proof, it is not hard to verify that the same result holds if $B$ is a finit union of intervals, $\operatorname{supp}(\mu) \subset B$ and $\mu^{\prime}>0$ a.e. on $B$. It is harder to prove, but still true that Lemma 3 remains valid if $\mu$ is a measure with compact support contained in $\mathbf{R}$ and $\mu \in \mathbf{R e g}$ (for the definitio see [8]). We limit ourselves to pointing out that a sufficien condition for $\mu \in \mathbf{R e g}$ is that

$$
\operatorname{cap}(\operatorname{supp}(\mu))=\operatorname{cap}\left(\left\{x \in \operatorname{supp}(\mu): \mu^{\prime}>0\right\}\right)
$$

(see Theorem 4.1.4 in [8]). If $\mu \in \mathbf{R e g}$ the only additional assumption to the ones imposed above in order that Theorem 1 (and its corollaries) take place is that $A \subset$ $\mathbf{C} \backslash \operatorname{conv}(\operatorname{supp}(\mu))$, where conv $(\operatorname{supp}(\mu))$ denotes the smallest interval containing $\operatorname{supp}(\mu)$.

\section{Applications to Quadratures}

Notice that (3) implies that $\left(\hat{\mu}-R_{n}\right) / A_{n}$ is holomorphic in $\mathbf{C} \backslash B$ and

$$
\frac{\hat{\mu}-R_{n}}{A_{n}}(z)=O\left(\frac{1}{z^{2 n-m(n)+1}}\right) \quad \text { as } \quad z \longrightarrow \infty .
$$

Consider the partial fraction decomposition of $R_{n}$

$$
R_{n}(z)=\sum_{i=1}^{N} \sum_{j=1}^{M_{j}} \frac{j ! A_{i, j}^{n}}{\left(z-x_{n, i}\right)^{j+1}} .
$$

$N$ denotes the total number of distinct poles of $R_{n}$. The points $x_{n, i}$ are zeros of $Q_{n} B_{n}$. Though the zeros of $Q_{n}$ are simple (see (2)) they may coincide with zeros of $B_{n}$; therefore, for given $x_{n, i}$ any value of $M_{j}$ is possible. Obviously, $N=N(n)$ and $M_{j}=M_{j}(n, i)$, but in order to simplify the notation, we omit the explicit reference to this dependence. 
Lemma 5. For any polynomial p of degree $\leq 2 n-m(n)-1$, we have

$$
\int \frac{p}{A_{n}}(x) d \mu(x)=\sum_{i=1}^{N} \sum_{j=1}^{M_{j}} A_{i, j}^{n}\left(\frac{p}{A) n}\right)^{(j)}\left(x_{n, i}\right) .
$$

Proof. If $p$ is a polynomial of degree $\leq 2 n-m(n)-1$, then due to (41), $p\left(\hat{\mu}-R_{n}\right) / A_{n}$ has a zero at infinit of order at least two and is holomorphic in $\mathbf{C} \backslash b$. Proceedings as in the proof of (2), and using (42), we obtain

$$
\begin{aligned}
0 & =\int_{\Gamma} \frac{p\left(\hat{\mu}-R_{n}\right)}{A_{n}}(z) d z=\int_{\Gamma} \frac{p \hat{\mu}}{A_{n}}(z) d z-\int_{\Gamma} \frac{p R_{n}}{A_{n}}(z) d z \\
& =2 \pi i\left[\int \frac{p}{A_{n}}(x) d \mu(x)-\sum_{i=1}^{n} \sum_{j=1}^{M_{j}} A_{i, j}^{n}\left(\frac{p}{A_{n}}\right)^{(j)}\left(x_{n, i}\right)\right] .
\end{aligned}
$$

This formula is equivalent to (43).

Let $f$ be a continuous function on $B$ such that the operations indicated in the following expressions have sense. Denote

$$
I(f)=\int f(x) d \mu(x), \quad I_{n}(f)=\sum_{i=1}^{N} \sum_{j=1}^{M_{j}} A_{i, j}^{n} f^{(j)}\left(x_{n, i}\right) .
$$

Formula (43) indicates that for any polynomial $p$ of degree $\leq 2 n-m(n)-1$

$$
I_{n}\left(\frac{p}{A_{n}}\right)=I\left(\frac{p}{A_{n}}\right) \text {. }
$$

As we show below, if $f$ is analytic on a neighborhood $V$ of $B$, then

$$
\lim _{n} I_{n}(f)=I(f) .
$$

From the definition of $I(f), I_{n}(f)$ and using arguments similar to those in the proofs of Lemmas 1 and 5, it is easy to verify

Lemma 6. Let $f \in H(V)$. Then, for any contour $\Gamma$ contained in $V$ such that $B \subset$ $\operatorname{Int}(\Gamma)$, we have

$$
I(f)-I_{n}(f)=\frac{1}{2 \pi i} \int_{\Gamma} f(z)\left(\hat{\mu}-R_{n}\right)(z) d z .
$$

Now, we can prove

Theorem 2. Let $f \in H(v)$. Under the conditions of Theorem 1, we have

$$
\varlimsup_{n}\left|I(f)-I_{n}(f)\right|^{1 / n} \leq \sup _{z \in \partial V} \exp \left\{(\theta-2)\left[\omega^{*}-V_{\lambda^{*}-\alpha}(z)\right]\right\} .
$$

Denote

$$
q_{n}(f)=\left|I(f)-I_{n}(f)\right|,
$$

then

$$
\sup _{f} \varlimsup_{n} q_{n}(f)^{1 / n}=\sup _{z \in \partial V} \exp \left\{(\theta-2)\left[\omega^{*}-V_{\lambda^{*}-\alpha}(z)\right]\right\},
$$

where $\operatorname{cap}(\partial V)>0$. 
Proof. Take $\Gamma$ as indicated in Lemma 6. From (44) and (25), it follows that

$$
\varlimsup_{n}\left|I(f)-I_{n}(f)\right|^{1 / n} \leq \sup _{z \in \Gamma} \exp \left\{(\theta-2)\left[\omega^{*}-V_{\lambda^{*}-\alpha}(z)\right]\right\} .
$$

We can take $\Gamma$ as close to $\partial V$ as we please; therefore, (45) immediately follows.

Since the right side of (45) does not depend on $f \in H(V)$, it follows that

$$
\sup _{f} \varlimsup_{n} q_{n}^{1 / n} \leq \sup _{z \in \partial V} \exp \left\{(\theta-2)\left[\omega^{*}-V_{\lambda^{*}-\alpha}(z)\right]\right\} .
$$

For the lower bound it is sufficien to notice that $f_{z}(x)=(z-x)^{-1}, z \in \mathbf{C} \backslash V$, belongs to $H(V)$, and

$$
I\left(f_{z}\right)-I_{n}\left(f_{z}\right)=\hat{\mu}(z)-R_{n}(z) .
$$

Therefore, by use of (25), we conclude with (46).

We leave to the reader the statement of the corresponding results when the conditions of Corollaries 1-4 are imposed.

Acknowledgment. The authors were partially supported by Research Grant 93-277 RG/Maths/LA from the Third World Academy of Science.

\section{References}

[1] A. AmBROLADZE, H. WALLIN (1998): Extremal polynomials with preassigned zeros and rational approximants. Const. Approx., 14:209 229.

[2] F. Cala, G. López (1995): From Padé to Padé-type approximants. Exact rate of convergence. Proc. Second Int. Conference on Approx. and Opt. in the Caribbean (M. Florenzano et al., eds.) Peter Lang Series in Approx. and Optimization, 8:155 163.

[3] A. A. Gonchar, G. LóPez (1978): On Markov's theorem for multipoint Padé approximation. Mat. Sb., 105(147):512 524. (English translation (1978): Math. USSR Sb., 34:449 459).

[4] A. A. Gonchar, E. A. Rakhmanov (1984): Equilibrium measures and the distribution of zeros of extremal polynomials. Mat. Sb., 125(167). (English translation (1986): Math USSR Sb., 53:119 130).

[5] L. KARLBERG, H. WALLIN (1990): Padé approximants and orthogonal polynomials for Markov Stieltjes functions. J. Comput. Appl. Math., 32:153 157.

[6] N. S. LANDKov (1972): Foundations of Modern Potential Theory. Grundlehren der Mathematischen Wissenschaften, vol. 190. Heidelberg: Springer-Verlag.

[7] G. LOPEZ, E. A. RAKHMANOV (1988): Rational approximation, orthogonal polynomials and equilibrium distributions. Proc. Second Int. Congress on Orthogonal Polynomials, Lecture Notes in Mathematics, vol. 1329. Heidelberg: Springer-Verlag.

[8] H. StaHL, V. TOTIK (1992): General Orthogonal Polynomials. Encyclopedia of Mathematics. New York: Cambridge University Press.

[9] J. L. WALSH (1969): Interpolation and Approximation by Rational Functions in the Complex Domain. Coll. Pub., Vol. XX. Providence, RI: American Mathematical Society.

\section{F. Cala Rodríguez}

Facultad de Matemáticas y Computación

Universidad de La Habana

Habana 4

Cuba
G. López Lagomasino

Departamento de Matemáticas

Universidad Carlos III de Madrid

c/Butarque 15

28911 Leganés

Spain 